\title{
Derivation of Bounds of an Integral Operator via Exponentially Convex Functions
}

\author{
Hong Ye, ${ }^{1}$ Ghulam Farid ${ }^{(D},{ }^{2}$ Babar Khan Bangash, ${ }^{2}$ and Lulu Cai ${ }^{1}$ \\ ${ }^{1}$ School of Electrical and Information Engineering, Quzhou University, Quzhou 324000, China \\ ${ }^{2}$ Department of Mathematics, COMSATS University Islamabad, Islamabad, Pakistan \\ Correspondence should be addressed to Lulu Cai; ysu-fbg@163.com
}

Received 17 March 2020; Accepted 21 May 2020; Published 4 July 2020

Academic Editor: Serkan Araci

Copyright (c) 2020 Hong Ye et al. This is an open access article distributed under the Creative Commons Attribution License, which permits unrestricted use, distribution, and reproduction in any medium, provided the original work is properly cited.

In this paper, bounds of fractional and conformable integral operators are established in a compact form. By using exponentially convex functions, certain bounds of these operators are derived and further used to prove their boundedness and continuity. A modulus inequality is established for a differentiable function whose derivative in absolute value is exponentially convex. Upper and lower bounds of these operators are obtained in the form of a Hadamard inequality. Some particular cases of main results are also studied.

\section{Introduction}

We start with the definition of convex function.

Definition 1 (see [1]). A function $f:[a, b] \longrightarrow \mathbb{R}$ is said to be convex if

$$
f(t x+(1-t) y) \leq t f(x)+(1-t) f(y),
$$

holds for all $x, y \in[a, b]$ and $t \in[0,1]$. If inequality (1) is reversed, then the function $f$ will be concave on $[a, b]$.

Convex functions are very useful in many areas of mathematics and other subjects due to their fascinating properties and characterizations. Their geometric and analytic interpretations provide straightforward proofs of many mathematical inequalities including Hadamard, Jensen, Hölder, and Minkowski [1-3]. Theoretically, convex functions have been generalized and extended as $h$-convex, $m$-convex, $s$-convex, $\quad(\alpha, m)$-convex, $\quad(h-m)$-convex, $(s, m)$-convex, etc. Awan et al. [4] defined the function named exponentially convex function as follows:

Definition 2. A function $f: K \subseteq \mathbb{R} \longrightarrow \mathbb{R}$, where $K$ is an interval, is said to be an exponentially convex function if

$$
f(t a+(1-t) b) \leq t \frac{f(a)}{e^{\alpha a}}+(1-t) \frac{f(b)}{e^{\alpha b}},
$$

holds for all $a, b \in K, t \in[0,1]$ and $\alpha \in \mathbb{R}$. If the inequality in (2) is reversed, then $f$ is called exponentially concave.

If $\alpha=0$, then (2) gives inequality (1). For some recent citations and utilization of exponentially convex functions, one can see [5-14] and references therein. Our goal in this paper is to prove generalized integral inequalities for exponentially convex functions by using integral operators given in Definition 7. In the following, we give definitions of Riemann-Liouville fractional integrals:

Definition 3. Let $f \in L_{1}[a, b]$. Then, the left-sided and rightsided Riemann-Liouville fractional integral operators of order $\mu \in \mathbb{C}(\mathscr{R}(\mu)>0)$ are defined by

$$
\begin{aligned}
& { }^{\mu} I_{a^{+}} f(x)=\frac{1}{\Gamma(\mu)} \int_{a}^{x}(x-t)^{\mu-1} f(t) \mathrm{d} t, \quad x>a, \\
& { }^{\mu} I_{b_{-}} f(x)=\frac{1}{\Gamma(\mu)} \int_{x}^{b}(t-x)^{\mu-1} f(t) \mathrm{d} t, \quad x<b .
\end{aligned}
$$

A $k$-fractional analogue is given as follows: 
Definition 4 (see [15]). Let $f \in L_{1}[a, b]$. Then, for $k>0$, the $k$-fractional integral operators of $f$ of order $\mu \in \mathbb{C}, \mathscr{R}(\mu)>0$ are defined by

$$
\begin{aligned}
& { }^{\mu} I_{a^{+}}^{k} f(x)=\frac{1}{k \Gamma_{\kappa}(\mu)} \int_{a}^{x}(x-t)^{(\mu / k)-1} f(t) \mathrm{d} t, \quad x>a, \\
& { }^{\mu} I_{b_{-}}^{k} f(x)=\frac{1}{k \Gamma_{k}(\mu)} \int_{x}^{b}(t-x)^{(\mu / k)-1} f(t) \mathrm{d} t, \quad x<b .
\end{aligned}
$$

A more general definition of the Riemann-Liouville fractional integral operators is given as follows:

Definition 5 (see [16]). Let $f:[a, b] \longrightarrow \mathbb{R}$ be an integrable function. Also, let $g$ be an increasing and positive function on $(a, b]$, having a continuous derivative $g^{\prime}$ on $(a, b)$. The left-sided and right-sided fractional integrals of a function $f$ with respect to another function $g$ on $[a, b]$ of order $\mu \in \mathbb{C}(\mathscr{R}(\mu)>0)$ are defined by

$$
{ }_{g}^{\mu} I_{a^{+}} f(x)=\frac{1}{\Gamma(\mu)} \int_{a}^{x}(g(x)-g(t))^{\mu-1} g^{\prime}(t) f(t) \mathrm{d} t, \quad x>a,
$$

${ }_{g}^{\mu} I_{b_{-}} f(x)=\frac{1}{\Gamma(\mu)} \int_{x}^{b}(g(t)-g(x))^{\mu-1} g^{\prime}(t) f(t) \mathrm{d} t, \quad x<b$,

where $\Gamma($.$) is the gamma function.$

Definition 6. Let $f:[a, b] \longrightarrow \mathbb{R}$ be an integrable function. Also, let $g$ be an increasing and positive function on $(a, b]$, having a continuous derivative $g^{\prime}$ on $(a, b)$. The leftsided and right-sided $k$-fractional integral operators, $k>0$, of a function $f$ with respect to another function $g$ on $[a, b]$ of order $\mu, k \in \mathbb{C}, \mathscr{R}(\mu)>0$ are defined by

$$
{ }_{g}^{\mu} I_{a^{+}}^{k} f(x)=\frac{1}{k \Gamma_{k}(\mu)} \int_{a}^{x}(g(x)-g(t))^{(\mu / k)-1} g^{\prime}(t) f(t) \mathrm{d} t, \quad x>a,
$$

${ }_{g}^{\mu} I_{b_{-}}^{k} f(x)=\frac{1}{k \Gamma_{k}(\mu)} \int_{x}^{b}(g(t)-g(x))^{(\mu / k)-1} g^{\prime}(t) f(t) \mathrm{d} t, \quad x<b$,

where $\Gamma_{k}($.$) is the k$-gamma function.

A compact form of integral operators defined above is given as follows:

Definition 7 (see [17]). Let $f, g:[a, b] \longrightarrow \mathbb{R}, 0<a<b$ be the functions such that $f$ be positive and $f \in L_{1}[a, b]$ and $g$ be differentiable and strictly increasing. Also, let $(\phi / x)$ be an increasing function on $[a, \infty)$. Then, for $x \in[a, b]$, the left- and right-sided integral operators are defined by

$$
\begin{aligned}
& \left(F_{a^{+}}^{\phi, g} f\right)(x)=\int_{a}^{x} K_{g}(x, t ; \phi) f(t) \mathrm{d}(g(t)), \quad x>a, \\
& \left(F_{b_{-}}^{\phi, g} f\right)(x)=\int_{x}^{b} K_{g}(t, x ; \phi) f(t) \mathrm{d}(g(t)), \quad x<b,
\end{aligned}
$$

where $K_{g}(x, y ; \phi)=(\phi(g(x)-g(y)) /(g(x)-g(y)))$.

Integral operators defined in (11) and (12) produce several fractional and conformable integral operators defined in $[16,18-25]$.

Remark 1. Integral operators given in (11) and (12) produce fractional and conformable integral operators as follows:

(i) If we consider $\phi(t)=\left(t^{\mu / k} / k \Gamma_{k}(\mu)\right)$, then (11) and (12) integral operators coincide with (9) and (10) fractional integral operators.

(ii) If we consider $\phi(t)=\left(t^{\mu} / \Gamma(\mu)\right), \mu>0$, then (11) and (12) integral operators coincide with (7) and (8) fractional integral operators.

(iii) If we consider $\phi(t)=\left(t^{\mu / k} / k \Gamma_{k}(\mu)\right)$ and $g$ as identity function (11) and (12), integral operators coincide with (5 and 6) fractional integral operators.

(iv) If we consider $\phi(t)=\left(t^{\mu} / \Gamma(\mu)\right), \mu>0$, and along with $g$ as identity function (11) and (12), integral operators coincide with (3 and 4) fractional integral operators.

(v) If we consider $\phi(t)=\left(t^{\mu / k} / k \Gamma_{k}(\mu)\right), k=1$, and $g(x)=\left(x^{\rho} / \rho\right), \rho>0$, then (11) and (12) produce Katugampola fractional integral operators defined by Chen and Katugampola in [18].

(vi) If we consider $\phi(t)=\left(t^{\mu / k} / k \Gamma_{k}(\mu)\right), k=1$, and $g(x)=\left(x^{\tau+s} /(\tau+s)\right), s>0$, then (11) and (12) produce generalized conformable integral operators defined by Khan and Khan in [22].

(vii) If we consider $\phi(t)=\left(t^{\mu / k} / k \Gamma_{k}(\mu)\right)$ and $g(x)=$ $\left((x-a)^{s} / s\right), s>0$, in (11) and $\phi(t)=\left(t^{\mu / k} / k \Gamma_{k}(\mu)\right)$ and $g(x)=-\left((b-x)^{s} / s\right), s>0$, in (12), respectively, then conformable $(k, s)$-fractional integrals are achieved as defined by Habib et al. in [20].

(viii) If we consider $\phi(t)=\left(t^{\mu / k} / k \Gamma_{k}(\mu)\right)$ and $g(x)=$ $\left(x^{1+s} /(1+s)\right)$, then (11) and (12) produce conformable fractional integrals defined by Sarikaya et al. in [24].

(ix) If we consider $\phi(t)=\left(t^{\mu / k} / k \Gamma_{k}(\mu)\right)$ and $g(x)=$ $\left((x-a)^{s} / s\right), s>0$, in (11) and $\phi(t)=\left(t^{\mu / k} / k \Gamma_{k}(\mu)\right)$ and $g(x)=-\left((b-x)^{s} / s\right), s>0$, in (12) with $k=1$, respectively, then conformable fractional integrals are achieved as defined by Jarad et al. in [21].

(x) If we consider $\phi(t)=t^{\mu / k} \mathscr{F}_{\rho, \lambda}^{\sigma, k}\left(w(t)^{\rho}\right)$, then (11) and (12) produce generalized $k$-fractional integral operators defined by Tunc et al. in [25].

(xi) If we consider $\phi(t)=(\exp (-A t) / \mu)$ and $A=((1-$ $\mu) / \mu), \mu>0$, then following generalized fractional integral operators with exponential kernel are obtained [19]: 


$$
\begin{aligned}
& { }_{g}^{\mu} E_{a^{+}} f(x)=\frac{1}{\mu} \int_{a}^{x} \exp \left(-\frac{1-\mu}{\mu}(g(x)-g(t))\right) f(t) \mathrm{d} t, \quad x>a, \\
& { }_{g}^{\mu} E_{b_{-}} f(x)=\frac{1}{\mu} \int_{x}^{b} \exp \left(-\frac{1-\mu}{\mu}(g(x)-g(t))\right) f(t) \mathrm{d} t, \quad x<b .
\end{aligned}
$$

(xii) If we consider $\phi(t)=\left(t^{\mu} / \Gamma(\mu)\right)$ and $g(t)=\ln t$, then Hadamard fractional integral operators will be obtained [16, 23].

(xiii) If we consider $\phi(t)=\left(t^{\mu} / \Gamma(\mu)\right)$ and $g(t)=-t^{-1}$, then Harmonic fractional integral operators given in [16] will be obtained and given as follows:

$$
\begin{aligned}
& { }^{\mu} R_{a^{+}} f(x)=\frac{t^{\mu}}{\Gamma(\mu)} \int_{a}^{x}(x-t)^{\mu-1} \frac{f(t)}{t^{\mu+1}} \mathrm{~d} t, \quad x>a, \\
& { }^{\mu} R_{b_{-}} f(x)=\frac{t^{\mu}}{\Gamma(\mu)} \int_{a}^{x}(t-x)^{\mu-1} \frac{f(t)}{t^{\mu+1}} \mathrm{~d} t, \quad x<b .
\end{aligned}
$$

(xiv) If we consider $\phi(t)=t^{\mu} \ln t$, then left- and rightsided logarithmic fractional integrals are obtained in [19] and given as follows:

$$
\begin{aligned}
{ }_{g}^{\mu} \mathscr{L}_{a^{+}} f(x)= & \int_{a}^{x}(g(x)-g(t))^{\mu-1} \ln (g(x) \\
& -g(t)) g^{\prime}(t) \mathrm{d} t, \quad x>a, \\
{ }^{\mu} \mathscr{L}_{b_{-}} f(x)= & \int_{a}^{x}(g(t)-g(x))^{\mu-1} \ln (g(x) \\
& -g(t)) g^{\prime}(t) \mathrm{d} t, \quad x<b .
\end{aligned}
$$

In the upcoming section, we will derive bounds of sum of the left- and right-sided integral operators defined in (11) and (12) for exponentially convex functions. These bounds lead to produce results associated to several kinds of wellknown operators for exponentially convex functions, some of the results are presented in particular cases. Further in Section 3, bounds are presented in the form of a Hadamard inequality; several Hadamard type inequalities are obtained.

\section{Bounds of Integral Operators and Their Consequences}

Theorem 1. Let $f:[a, b] \longrightarrow \mathbb{R}$ be a positive and exponentially convex function and $g:[a, b] \longrightarrow \mathbb{R}$ be a differentiable and strictly increasing function. Also, let $(\phi / x)$ be an increasing function on $[a, b]$. Then, for $x \in[a, b]$ the following inequality for integral operators (11) and (12) holds

$$
\begin{aligned}
& \left(F_{a^{+}}^{\phi, g} f\right)(x)+\left(F_{b^{-}}^{\phi, g} f\right)(x) \\
& \leq \phi(g(x)-g(a))\left(\frac{f(x)}{e^{\alpha x}}+\frac{f(a)}{e^{\alpha a}}\right)+\phi(g(b) \\
& \quad-g(x))\left(\frac{f(x)}{e^{\alpha x}}+\frac{f(b)}{e^{\alpha b}}\right) .
\end{aligned}
$$

Proof. For the kernel of integral operator (11), we have $K_{g}(x, t ; \phi) g^{\prime}(t) \leq K_{g}(x, a ; \phi) g^{\prime}(t), \quad x \in(a, b]$ and $t \in[a, x)$.

An exponentially convex function satisfies the following inequality:

$$
f(t) \leq\left(\frac{x-t}{x-a}\right) \frac{f(a)}{e^{\alpha a}}+\left(\frac{t-a}{x-a}\right) \frac{f(x)}{e^{\alpha x}} .
$$

Inequalities (17) and (18) lead to the following integral inequality:

$$
\begin{aligned}
\int_{a}^{x} K_{g}(x, t ; \phi) g^{\prime}(t) f(t) \mathrm{d} t \\
\leq K_{g}(x, a ; \phi)\left(\frac{f(a)}{e^{\alpha a}} \int_{a}^{x}\left(\frac{x-t}{x-a}\right) g^{\prime}(t) \mathrm{d} t\right. \\
\left.\quad+\frac{f(x)}{e^{\alpha x}} \int_{a}^{x}\left(\frac{t-a}{x-a}\right) g^{\prime}(t) \mathrm{d} t\right)
\end{aligned}
$$

while (19) gives

$$
\left(F_{a^{+}}^{\phi, g} f\right)(x) \leq \phi(g(x)-g(a))\left(\frac{f(x)}{e^{\alpha x}}+\frac{f(a)}{e^{\alpha a}}\right) .
$$

Again, for the kernel of integral operator (12), we have $K_{g}(t, x ; \phi) g^{\prime}(t) \leq K_{g}(b, x ; \phi) g^{\prime}(t), \quad t \in(x, b]$ and $x \in[a, b)$.

An exponentially convex function satisfies the following inequality:

$$
f(t) \leq\left(\frac{t-x}{b-x}\right) \frac{f(b)}{e^{\alpha b}}+\left(\frac{b-t}{b-x}\right) \frac{f(x)}{e^{\alpha x}} .
$$

Inequalities (21) and (22) lead to the following integral inequality:

$$
\begin{aligned}
& \int_{x}^{b} K_{g}(t, x ; \phi) g^{\prime}(t) f(t) \mathrm{d} t \\
& \quad \leq K_{g}(b, x ; \phi)\left(\frac{f(b)}{e^{\alpha b}} \int_{x}^{b}\left(\frac{t-x}{b-x}\right) g^{\prime}(t) \mathrm{d} t\right. \\
& \left.+\frac{f(x)}{e^{\alpha x}} \int_{x}^{b}\left(\frac{b-t}{b-x}\right) g^{\prime}(t) \mathrm{d} t\right)
\end{aligned}
$$

while (23) gives

$$
\left(F_{b^{-}}^{\phi, g} f\right)(x) \leq \phi(g(b)-g(x))\left(\frac{f(x)}{e^{\alpha x}}+\frac{f(b)}{e^{\alpha b}}\right) .
$$

By adding (20) and (24), (16) can be achieved.

The following remark connected the abovementioned theorem with already known results.

\section{Remark 2}

(1) For $\phi(t)=\left(t^{\mu} / \Gamma(\mu)\right), \mu>0$, and $\alpha=0$ in (16), Corollary 1 in [26] can be achieved.

(2) For $\phi(t)=\left(t^{\mu} / \Gamma(\mu)\right), \mu>0, g(x)=x$, and $\alpha=0$ in (16), Corollary 1 in [27] can be achieved. 
(3) For $\alpha=0$, in (16), Theorem 1 in [28] can be achieved.

Next results indicate upper bounds of several known fractional and conformable integral operators.

Proposition 1. Let $\phi(t)=\left(t^{\mu} / \Gamma(\mu)\right), \mu>0$. Then, (11) and (12) produce the fractional integral operators (7) and (8) as follows:

$$
\begin{aligned}
& \left(F_{a^{+}}^{\left(t^{\mu} / \Gamma(\mu)\right), g} f\right)(x):={ }_{g}^{\mu} I_{a^{+}} f(x), \\
& \left(F_{b^{-}}^{\left(t^{\mu} / \Gamma(\mu)\right), g} f\right)(x):={ }_{g}^{\mu} I_{b^{-}} f(x) .
\end{aligned}
$$

Further they satisfy the following bound for $\mu \geq 1$ :

$$
\begin{aligned}
\left({ }_{g}^{\mu} I_{a^{+}} f\right)(x)+\left({ }_{g}^{\mu} I_{b^{-}} f\right)(x) & \\
\leq & \frac{(g(x)-g(a))^{\mu}}{\Gamma(\mu)}\left(\frac{f(x)}{e^{\alpha x}}+\frac{f(a)}{e^{\alpha a}}\right) \\
& +\frac{(g(b)-g(x))^{\mu}}{\Gamma(\mu)}\left(\frac{f(x)}{e^{\alpha x}}+\frac{f(b)}{e^{\alpha b}}\right) .
\end{aligned}
$$

Proposition 2. Let $g(x)=I(x)=x$. Then, (11) and (12) produce integral operators defined in [29] as follows:

$$
\begin{aligned}
& \left(F_{a^{+}}^{\phi, I} f\right)(x):=\left({ }_{a^{+}} I_{\phi} f\right)(x)=\int_{a}^{x} \frac{\phi(x-t)}{(x-t)} f(t) \mathrm{d} t, \\
& \left(F_{b^{-}}^{\phi, I} f\right)(x):=\left({ }_{b^{-}} I_{\phi} f\right)(x)=\int_{x}^{b} \frac{\phi(t-x)}{(t-x)} f(t) \mathrm{d} t .
\end{aligned}
$$

Further they satisfy the following bound:

$$
\begin{aligned}
& \left({ }_{a^{+}} I_{\phi} f\right)(x)+\left({ }_{b^{-}} I_{\phi} f\right)(x) \\
& \quad \leq \phi(x-a)\left(\frac{f(x)}{e^{\alpha x}}+\frac{f(a)}{e^{\alpha a}}\right)+\phi(b-x)\left(\frac{f(x)}{e^{\alpha x}}+\frac{f(b)}{e^{\alpha b}}\right) .
\end{aligned}
$$

Corollary 1. If we take $\phi(t)=\left(t^{\mu / k} / k \Gamma_{k}(\mu)\right)$, then (11) and (12) produce the fractional integral operators (9) and (10) as follows:

$$
\begin{aligned}
& \left(F_{a^{+}}^{\left(t^{\mu / k} / k \Gamma_{k}(\mu)\right), g} f\right)(x):={ }_{g}^{\mu} I_{a^{+}}^{k} f(x), \\
& \left(F_{b^{-}}^{\left(t^{\mu / k} / k \Gamma_{k}(\mu)\right), g} f\right)(x):={ }_{g}^{\mu} I_{b^{-}}^{k} f(x) .
\end{aligned}
$$

From (16), the following bound holds for $\mu \geq k$ :

$$
\begin{aligned}
\left({ }_{g}^{\mu} I_{a^{+}}^{k} f\right)(x)+\left({ }_{g}^{\mu} I_{b^{-}}^{k} f\right)(x) \leq & \frac{(g(x)-g(a))^{\mu / k}}{k \Gamma_{k}(\mu)}\left(\frac{f(x)}{e^{\alpha x}}+\frac{f(a)}{e^{\alpha a}}\right) \\
& +\frac{(g(b)-g(x))^{\mu / k}}{k \Gamma_{k}(\mu)}\left(\frac{f(x)}{e^{\alpha x}}+\frac{f(b)}{e^{\alpha b}}\right) .
\end{aligned}
$$

Corollary 2. If we take $\phi(t)=\left(t_{\mu} / \Gamma(\mu)\right), \mu>0$, and $g(x)=I(x)=x$, then (11) and (12) produce left- and rightsided Riemann-Liouville fractional integral operators (3) and (4) as follows:

$$
\begin{aligned}
& \left(F_{a^{+}}^{\left(t^{\mu} / \Gamma(\mu)\right), I} f\right)(x):={ }^{\mu} I_{a^{+}} f(x), \\
& \left(F_{b^{-}}^{\left(t^{\mu} / \Gamma(\mu)\right), I} f\right)(x):={ }^{\mu} I_{b^{-}} f(x) .
\end{aligned}
$$

From (16), the following bound holds for $\mu \geq 1$ :

$$
\begin{aligned}
& \left({ }^{\mu} I_{a^{+}} f\right)(x)+\left({ }^{\mu} I_{b^{-}} f\right)(x) \\
& \quad \leq \frac{(x-a)^{\mu}}{\Gamma(\mu)}\left(\frac{f(x)}{e^{\alpha x}}+\frac{f(a)}{e^{\alpha a}}\right)+\frac{(b-x)^{\mu}}{\Gamma(\mu)}\left(\frac{f(x)}{e^{\alpha x}}+\frac{f(b)}{e^{\alpha b}}\right) .
\end{aligned}
$$

Corollary 3. If we take $\phi(t)=\left(t^{\mu / k} / k \Gamma_{k}(\mu)\right)$ and $g(x)=I(x)=x$, then (11) and (12) produce the fractional integral operators (5) and (6) as follows:

$$
\begin{aligned}
& \left(F_{a^{+}}^{\left(t^{\mu / k} / k \Gamma_{k}(\mu)\right), I} f\right)(x):={ }^{\mu} I_{a^{+}}^{k} f(x), \\
& \left(F_{b^{-}}^{\left(t^{\mu / k} / k \Gamma_{k}(\mu)\right), I} f\right)(x):={ }^{\mu} I_{b^{-}}^{k} f(x) .
\end{aligned}
$$

From (16), the following bound holds for $\mu \geq k$ :

$$
\begin{aligned}
& \left({ }^{\mu} I_{a^{+}}^{k} f\right)(x)+\left({ }^{\mu} I_{b^{-}}^{k} f\right)(x) \\
& \quad \leq \frac{(x-a)^{\mu / k}}{k \Gamma_{k}(\mu)}\left(\frac{f(x)}{e^{\alpha x}}+\frac{f(a)}{e^{\alpha a}}\right)+\frac{(b-x)^{\mu / k}}{k \Gamma_{k}(\mu)}\left(\frac{f(x)}{e^{\alpha x}}+\frac{f(b)}{e^{\alpha b}}\right) .
\end{aligned}
$$

Corollary 4. If we take $\phi(t)=\left(t^{\mu} / \Gamma(\mu)\right), \mu>0$ and $g(x)=\left(x^{\rho} / \rho\right), \rho>0$, then (11) and (12) produce the fractional integral operators defined in [18] as follows:

$$
\begin{aligned}
\left(F_{a^{+}}^{\left(t^{\mu} / \Gamma(\mu)\right), g} f\right)(x) & =\left({ }^{\rho} I_{a^{+}}^{\mu} f\right)(x) \\
& =\frac{\rho^{1-\mu}}{\Gamma(\mu)} \int_{a}^{x}\left(x^{\rho}-t^{\rho}\right)^{\mu-1} t^{\rho-1} f(t) \mathrm{d} t \\
\left(F_{b^{-}}^{\left(t^{\mu} / \Gamma(\mu)\right), g} f\right)(x) & =\left({ }^{\rho} I_{b^{-}}^{\mu} f\right)(x) \\
& =\frac{\rho^{1-\mu}}{\Gamma(\mu)} \int_{x}^{b}\left(t^{\rho}-x^{\rho}\right)^{\mu-1} t^{\rho-1} f(t) \mathrm{d} t
\end{aligned}
$$

From (16), they satisfy the following bound:

$$
\begin{aligned}
& \left({ }^{\rho} I_{a^{+}}^{\mu} f\right)(x)+\left({ }^{\rho} I_{b^{-}}^{\mu} f\right)(x) \\
& \quad \leq \frac{\left(x^{\rho}-a^{\rho}\right)^{\mu}}{\Gamma(\mu)\left(\rho^{\mu}\right)}\left(\frac{f(x)}{e^{\alpha x}}+\frac{f(a)}{e^{\alpha a}}\right)+\frac{\left(b^{\rho}-x^{\rho}\right)^{\mu}}{\Gamma(\mu)\left(\rho^{\mu}\right)}\left(\frac{f(x)}{e^{\alpha x}}+\frac{f(b)}{e^{\alpha b}}\right) .
\end{aligned}
$$


Corollary 5. If we take $\phi(t)=\left(t^{\mu} / \Gamma(\mu)\right), \mu>0$, and $g(x)=\left(x^{n+1} /(n+1)\right), n>0$, then (11) and (12) produce the fractional integral operators defined as follows:

$$
\begin{aligned}
\left(F_{a^{+}}^{\left(t^{\mu} / \Gamma(\mu)\right), g} f\right)(x) & =\left({ }^{n} I_{a^{+}}^{\mu} f\right)(x) \\
& =\frac{(n+1)^{1-\mu}}{\Gamma(\mu)} \int_{a}^{x}\left(x^{n+1}-t^{n+1}\right)^{\mu-1} t^{n} f(t) \mathrm{d} t, \\
\left(F_{b^{-}}^{\left(t^{\mu} / \Gamma(\mu)\right), g} f\right)(x) & =\left({ }^{n} I_{b^{-}}^{\mu} f\right)(x) \\
& =\frac{(n+1)^{1-\mu}}{\Gamma(\mu)} \int_{x}^{b}\left(t^{n+1}-x^{n+1}\right)^{\mu-1} t^{n} f(t) \mathrm{d} t .
\end{aligned}
$$

From (16), they satisfy the following bound:

$$
\begin{aligned}
& \left({ }^{n} I_{a^{+}}^{\mu} f\right)(x)+\left({ }^{n} I_{b^{-}}^{\mu} f\right)(x) \\
& \leq \frac{\left(x^{n+1}-a^{n+1}\right)^{\mu}}{\Gamma(\mu)(n+1)^{\mu}}\left(\frac{f(x)}{e^{\alpha x}}+\frac{f(a)}{e^{\alpha a}}\right) \\
& \quad+\frac{\left(x^{n+1}-a^{n+1}\right)^{\mu}}{\Gamma(\mu)(n+1)^{\mu}}\left(\frac{f(x)}{e^{\alpha x}}+\frac{f(b)}{e^{\alpha b}}\right) .
\end{aligned}
$$

Next, we prove boundedness and continuity of integral operators.

Theorem 2. Let the assumptions of Theorem 1 are satisfied. If $f \in L_{\infty}[a, b]$, then integral operators defined in (11) and (12) are continuous.

Proof. From (20), we have

$$
\left(F_{a^{+}}^{\phi, g} f\right)(x) \leq \phi(g(x)-g(a))\|f\|_{\infty}\left(\frac{1}{e^{\alpha x}}+\frac{1}{e^{\alpha a}}\right) .
$$

It is given that $(\phi / x)$ is increasing on $[a, b]$ :

$$
\begin{aligned}
& \frac{\phi(g(x)-g(a))}{g(x)-g(a)} \leq \frac{\phi(g(b)-g(a))}{g(b)-g(a)}, \\
& \phi(g(x)-g(a)) \leq \frac{\phi(g(b)-g(a)) g(x)-g(a)}{g(b)-g(a)} .
\end{aligned}
$$

Further $g$ is increasing; therefore, we have

$$
\phi(g(x)-g(a)) \leq \phi(g(b)-g(a)) .
$$

Therefore, (39) gives

$$
\left(F_{a^{+}}^{\phi, g} f\right)(x) \leq \phi(g(b)-g(a))\|f\|_{\infty}\left(e^{-\alpha x}+e^{-\alpha a}\right) .
$$

If $\alpha>0$, then $e^{-\alpha x}$ is decreasing on $[a, b]$ and we get

$$
\left(F_{a^{+}}^{\phi, g} f\right)(x) \leq 2 e^{-\alpha a} \phi(g(b)-g(a))\|f\|_{\infty} .
$$
(42)

If $\alpha<0$, then $e^{-\alpha x}$ is increasing on $[a, b]$ and we get from

$$
\left(F_{a^{+}}^{\phi, g} f\right)(x) \leq 2 e^{-\alpha b} \phi(g(b)-g(a))\|f\|_{\infty} .
$$

Hence, $\left(F_{a^{+}}^{\phi, g} f\right)(x)$ is bounded and it is linear, and therefore, $\left(F_{a^{+}}^{\phi, g} f\right)(x)$ is continuous.

Similarly, continuity of $\left(F_{b^{-}}^{\phi, g} f\right)(x)$ can be proved.

For a differentiable function $f$, as $\left|f^{\prime}\right|$ is exponentially convex, the following result holds:

Theorem 3. Let $f: I \longrightarrow \mathbb{R}$ be a differentiable function. If $\left|f^{\prime}\right|$ is exponentially convex and $g: I \longrightarrow \mathbb{R}$ is a differentiable and strictly increasing function. Also, let $(\phi / x)$ be an increasing function on $I$, then for $a, b \in I, a<b$, the following inequalities for integral operators holds:

$$
\left|F_{a^{+}}^{\phi, g}(f * g)(x)\right| \leq \phi(g(x)-g(a))\left(\frac{\left|f^{\prime}(a)\right|}{e^{\alpha a}}+\frac{\left|f^{\prime}(x)\right|}{e^{\alpha x}}\right),
$$

$$
\left|F_{b^{-}}^{\phi, g}(f * g)(x)\right| \leq \phi(g(b)-g(x))\left(\frac{\left|f^{\prime}(b)\right|}{e^{\alpha b}}+\frac{\left|f^{\prime}(x)\right|}{e^{\alpha x}}\right),
$$

where

$$
\begin{aligned}
& F_{a^{+}}^{\phi, g}(f * g)(x)=\int_{a}^{x} K_{g}(x, t ; \phi) g^{\prime}(t) f^{\prime}(t) \mathrm{d} t, \\
& F_{b^{-}}^{\phi, g}(f * g)(x)=\int_{x}^{b} K_{g}(t, x ; \phi) g^{\prime}(t) f^{\prime}(t) \mathrm{d} t .
\end{aligned}
$$

Proof. An exponentially convex function $\left|f^{\prime}\right|$ satisfies the following inequality:

$$
\left|f^{\prime}(t)\right| \leq\left(\frac{x-t}{x-a}\right) \frac{\left|f^{\prime}(a)\right|}{e^{\alpha a}}+\left(\frac{t-a}{x-a}\right) \frac{\left|f^{\prime}(x)\right|}{e^{\alpha x}} .
$$

From which, we can write

$$
f^{\prime}(t) \leq\left(\frac{x-t}{x-a}\right) \frac{\left|f^{\prime}(a)\right|}{e^{\alpha a}}+\left(\frac{t-a}{x-a}\right) \frac{\left|f^{\prime}(x)\right|}{e^{\alpha x}} .
$$

Inequalities (17) and (49) lead to the following integral inequality:

$$
\begin{aligned}
& \int_{a}^{x} K_{g}(x, t ; \phi) g^{\prime}(t) f^{\prime}(t) \mathrm{d} t \\
& \leq K_{g}(x, a ; \phi)\left(\frac{\left|f^{\prime}(a)\right|}{e^{\alpha a}} \int_{a}^{x}\left(\frac{x-t}{x-a}\right) g^{\prime}(t) \mathrm{d} t\right. \\
& \left.\quad+\left(\frac{\left|f^{\prime}(x)\right|}{e^{\alpha x}}\right) \int_{a}^{x}\left(\frac{t-a}{x-a}\right) g^{\prime}(t) \mathrm{d} t\right),
\end{aligned}
$$

while (50) gives

$$
F_{a^{+}}^{\phi, g}(f * g)(x) \leq \phi(g(x)-g(a))\left[\left(\frac{\left|f^{\prime}(a)\right|}{e^{\alpha a}}+\frac{\left|f^{\prime}(x)\right|}{e^{\alpha x}}\right)\right] .
$$

From (48), we can write

$$
f^{\prime}(t) \geq-\left(\left(\frac{x-t}{x-a}\right) \frac{\left|f^{\prime}(a)\right|}{e^{\alpha a}}+\left(\frac{t-a}{x-a}\right) \frac{\left|f^{\prime}(x)\right|}{e^{\alpha x}}\right) .
$$


Adopting the same method as we did for (49), the following integral inequality holds:

$$
F_{a^{+}}^{\phi, g}(f * g)(x) \geq-\phi(g(x)-g(a))\left(\frac{\left|f^{\prime}(a)\right|}{e^{\alpha a}}+\frac{\left|f^{\prime}(x)\right|}{e^{\alpha x}}\right) .
$$

From (51) and (53), (45) can be achieved.

An exponentially convex function $\left|f^{\prime}\right|$ satisfies the following inequality:

$$
\left|f^{\prime}(t)\right| \leq\left(\frac{t-x}{b-x}\right) \frac{\left|f^{\prime}(b)\right|}{e^{\alpha b}}+\left(\frac{b-t}{b-x}\right) \frac{\left|f^{\prime}(x)\right|}{e^{\alpha x}} .
$$

From which, we can write

$$
f^{\prime}(t) \leq\left(\frac{t-x}{b-x}\right) \frac{\left|f^{\prime}(b)\right|}{e^{\alpha b}}+\left(\frac{b-t}{b-x}\right) \frac{\left|f^{\prime}(x)\right|}{e^{\alpha x}} .
$$

Inequalities (21) and (55) lead the following integral inequality:

$$
\begin{aligned}
& \int_{x}^{b} K_{g}(t, x ; \phi) g^{\prime}(t) f^{\prime}(t) \mathrm{d} t \\
& \leq K_{g}(b, x ; \phi)\left(\frac{\left|f^{\prime}(b)\right|}{e^{\alpha b}} \int_{x}^{b}\left(\frac{x-t}{b-x}\right) g^{\prime}(t) \mathrm{d} t\right. \\
& \left.\quad+\frac{\left|f^{\prime}(x)\right|}{e^{\alpha x}} \int_{x}^{b}\left(\frac{b-t}{b-x}\right) g^{\prime}(t) \mathrm{d} t\right),
\end{aligned}
$$

while (56) gives

$$
F_{b^{-}}^{\phi, g}(f * g)(x) \leq \phi(g(b)-g(x))\left(\frac{\left|f^{\prime}(b)\right|}{e^{\alpha b}}+\frac{\left|f^{\prime}(x)\right|}{e^{\alpha x}}\right) .
$$

From (54), we can write

$$
f^{\prime}(t) \geq-\left(\left(\frac{t-x}{b-x}\right) \frac{\left|f^{\prime}(b)\right|}{e^{\alpha b}}+\left(\frac{b-t}{b-x}\right) \frac{\left|f^{\prime}(x)\right|}{e^{\alpha x}}\right) .
$$

Adopting the same method as we did for (55), the following inequality holds:

$$
F_{b^{-}}^{\phi, g}(f * g)(x) \geq-\phi(g(b)-g(x))\left(\frac{\left|f^{\prime}(b)\right|}{e^{\alpha b}}+\frac{\left|f^{\prime}(x)\right|}{e^{\alpha x}}\right) .
$$

From (57) and (59), (46) can be achieved.

\section{Hadamard Type Inequalities for Exponentially Convex Function}

In this section, we prove the Hadamard type inequality for an exponentially convex function. In order to prove this inequality result, we need the following lemma.

Lemma 1 (see [30]). Let $f:[a, b] \longrightarrow \mathbb{R}$ be an exponentially convex function. If $f$ is exponentially symmetric, then the following inequality holds:

$$
f\left(\frac{a+b}{2}\right) \leq \frac{f(x)}{e^{\alpha x}}, \quad x \in[a, b] .
$$

Theorem 4. Let $f:[a, b] \longrightarrow \mathbb{R}$ be positive, exponentially convex, and symmetric about $((a+b) / 2)$ and $g:[a, b]$ $\longrightarrow \mathbb{R}$ be a differentiable and strictly increasing function. Also, let $(\phi / x)$ be an increasing function on $[a, b]$. Then, for $a, b \in I, a<b$, the following estimations of Hadamard type are valid.

$$
\begin{aligned}
& h(\alpha) f\left(\frac{a+b}{2}\right)\left(\left(F_{b^{-}}^{\phi, g}(1)\right)(a)+\left(F_{a^{+}}^{\phi, g}(1)\right)(b)\right) \\
& \quad \leq\left(\left(F_{b^{-}}^{\phi, g} f\right)(a)+\left(F_{a^{+}}^{\phi, g} f\right)(b)\right) \\
& \quad \leq 2 \phi(g(b)-g(a))\left(\frac{f(b)}{e^{\alpha b}}+\frac{f(a)}{e^{\alpha a}}\right),
\end{aligned}
$$

where $h(\alpha)=e^{\alpha b}$ for $\alpha<0$ and $h(\alpha)=e^{\alpha a}$ for $\alpha \geq 0$.

Proof. For the kernel of integral operator (11), we have

$$
K_{g}(x, a ; \phi) g^{\prime}(x) \leq K_{g}(b, a ; \phi) g^{\prime}(x), \quad x \in(a, b] .
$$

An exponentially convex function satisfies the following inequality:

$$
f(x) \leq\left(\frac{x-a}{b-a}\right) \frac{f(b)}{e^{\alpha b}}+\left(\frac{b-x}{b-a}\right) \frac{f(a)}{e^{\alpha a}} .
$$

Inequalities (62) and (63) lead the following integral inequality:

$$
\begin{aligned}
& \int_{a}^{b} K_{g}(x, a ; \phi) g^{\prime}(x) f(x) \mathrm{d} x \\
& \leq K_{g}(b, a ; \phi)\left(\frac{f(b)}{e^{\alpha b}} \int_{a}^{b}\left(\frac{x-a}{b-a}\right) g^{\prime}(x) \mathrm{d} x\right. \\
& \left.+\frac{f(a)}{e^{\alpha a}} \int_{a}^{b}\left(\frac{b-x}{b-a}\right) g^{\prime}(x) \mathrm{d} x\right),
\end{aligned}
$$

while (64) gives

$$
\left(F_{b^{-}}^{\phi, g} f\right)(a) \leq \phi(g(b)-g(a))\left(\frac{f(b)}{e^{\alpha b}}+\frac{f(a)}{e^{\alpha a}}\right) .
$$

On the contrary, for the kernel of integral operator (12), we have

$$
K_{g}(b, x ; \phi) g^{\prime}(x) \leq K_{g}(b, a ; \phi) g^{\prime}(x) .
$$

Inequalities (63) and (66) lead the following integral inequality: 


$$
\begin{aligned}
& \int_{a}^{b} K_{g}(b, x ; \phi) g^{\prime}(x) f(x) \mathrm{d} x \\
& \leq K_{g}(b, a ; \phi)\left(\frac{f(b)}{e^{\alpha b}} \int_{a}^{b}\left(\frac{x-a}{b-a}\right) g^{\prime}(x) \mathrm{d} x\right. \\
& \left.\quad+\frac{f(a)}{e^{\alpha a}} \int_{a}^{b}\left(\frac{b-x}{b-a}\right) g^{\prime}(x) \mathrm{d} x\right),
\end{aligned}
$$

while the abovementioned inequality gives

$$
\left(F_{a^{+}}^{\phi, g} f\right)(b) \leq \phi(g(b)-g(a))\left(\frac{f(b)}{e^{\alpha b}}+\frac{f(a)}{e^{\alpha a}}\right) .
$$

From (65) and (68), the following inequality can be obtained:

$$
\left(F_{a^{+}}^{\phi, g} f\right)(b)+\left(F_{b^{-}}^{\phi, g} f\right)(a) \leq 2 \phi(g(b)-g(a))\left(\frac{f(b)}{e^{\alpha b}}+\frac{f(a)}{e^{\alpha a}}\right) .
$$

Now, using Lemma 1 and multiplying (60) with $K_{g}(x, a ; \phi) g^{\prime}(x)$, then integrating over $[a, b]$, we have

$$
\begin{aligned}
& \int_{a}^{b} K_{g}(x, a ; \phi) f\left(\frac{a+b}{2}\right) g^{\prime}(x) d x \\
& \quad \leq \int_{a}^{b} \frac{1}{e^{\alpha x}} K_{g}(x, a ; \phi) g^{\prime}(x) f(x) \mathrm{d} x .
\end{aligned}
$$

From which, we have

$$
f\left(\frac{a+b}{2}\right)\left(F_{b^{-}}^{\phi, g}(1)\right)(a) \leq \frac{1}{h(\alpha)}\left(F_{b^{-}}^{\phi, g} f\right)(a) .
$$

Again using Lemma 1 and multiplying (60) with $K_{g}(b, x ; \phi) g^{\prime}(x)$, then integrating over $[a, b]$, we have

$$
\begin{aligned}
& \int_{a}^{b} K_{g}(b, x ; \phi) f\left(\frac{a+b}{2}\right) g^{\prime}(x) \mathrm{d} x \\
& \quad \leq \int_{a}^{b} \frac{1}{e^{\alpha x}} K_{g}(b, x ; \phi) g^{\prime}(x) f(x) \mathrm{d} x .
\end{aligned}
$$

From which, we have

$$
f\left(\frac{a+b}{2}\right)\left(F_{a^{+}}^{\phi, g}(1)\right)(b) \leq \frac{1}{h(\alpha)}\left(F_{a^{+}}^{\phi, g} f\right)(b) .
$$

From (71) and (73), the following inequality can be achieved:

$$
\begin{gathered}
f\left(\frac{a+b}{2}\right)\left(F_{b^{-}}^{\phi, g}(1)\right)(a)+\left(F_{a^{+}}^{\phi, g}(1)\right)(b) \\
\quad \leq \frac{1}{h(\alpha)}\left(\left(F_{b^{-}}^{\phi, g} f\right)(a)+\left(F_{a^{+}}^{\phi, g} f\right)(b)\right) .
\end{gathered}
$$

From (69) and (74), (61) can be achieved.

Remark 3. For $\alpha=0$, in (61), Theorem 3 in [28] can be achieved.
Corollary 6. If we put $\phi(t)=\left(t^{\mu / k} / k \Gamma_{k}(\mu)\right)$, then the inequality (61) produces the following Hadamard type inequality:

$$
\begin{aligned}
& h(\alpha) f\left(\frac{a+b}{2}\right)\left({ }_{g}^{\mu} I_{b_{-}}^{k}(1)(a)+{ }_{g}^{\mu} I_{a^{+}}^{k}(1)(b)\right) \\
& \leq\left({ }_{g}^{\mu} I_{b_{-}}^{k} f(a)+{ }_{g}^{\mu} I_{a^{+}}^{k} f(b)\right) \\
& \leq \frac{2(g(b)-g(a))^{\mu / k}}{\left(k \Gamma_{k}(\mu)\right)}\left(\frac{f(b)}{e^{\alpha b}}+\frac{f(a)}{e^{\alpha a}}\right) .
\end{aligned}
$$

Corollary 7. If we put $\phi(t)=\left(t^{\mu} / \Gamma(\mu)\right)$, then the inequality (61) produces the following Hadamard type inequality:

$$
\begin{aligned}
& h(\alpha) f\left(\frac{a+b}{2}\right)\left({ }_{g}^{\mu} I_{b_{-}}(1)(a)+{ }_{g}^{\mu} I_{a^{+}}(1)(b)\right) \\
& \leq\left({ }_{g}^{\mu} I_{b_{-}} f(a)+{ }_{g}^{\mu} I_{a^{+}} f(b)\right) \\
& \leq \frac{2(g(b)-g(a))^{\mu}}{\Gamma(\mu)}\left(\frac{f(b)}{e^{\alpha b}}+\frac{f(a)}{e^{\alpha a}}\right) .
\end{aligned}
$$

Corollary 8. If we put $\phi(t)=\left(t^{\mu / k} / k \Gamma_{k}(\mu)\right)$ and $g$ as identity function, then the inequality (61) produces the following Hadamard type inequality:

$$
\begin{aligned}
& h(\alpha) f\left(\frac{a+b}{2}\right)\left({ }^{\mu} I_{b_{-}}^{k}(1)(a)+{ }^{\mu} I_{a^{+}}^{k}(1)(b)\right) \\
& \leq\left({ }^{\mu} I_{b_{-}}^{k} f(a)+{ }^{\mu} I_{a^{+}}^{k} f(b)\right) \\
& \quad \leq \frac{2(b-a)^{\mu / k}}{k \Gamma_{k}(\mu)}\left(\frac{f(b)}{e^{\alpha b}}+\frac{f(a)}{e^{\alpha a}}\right) .
\end{aligned}
$$

Corollary 9. If we put $\phi(t)=\left(t^{\mu} / \Gamma(\mu)\right)$ and $g$ as identity function, then the inequality (61) produces the following Hadamard type inequality:

$$
\begin{aligned}
& h(\alpha) f\left(\frac{a+b}{2}\right)\left({ }^{\mu} I_{b_{-}}(1)(a)+{ }^{\mu} I_{a^{+}}(1)(b)\right) \\
& \leq\left({ }^{\mu} I_{b_{-}} f(a)+{ }^{\mu} I_{a^{+}} f(b)\right) \\
& \leq \frac{2(b-a)}{\Gamma(\mu)}\left(\frac{f(b)}{e^{\alpha b}}+\frac{f(a)}{e^{\alpha a}}\right) .
\end{aligned}
$$

\section{Concluding Remarks}

We have studied an integral operator for exponentially convex functions; this operator has direct consequences to several fractional and conformable integral operators. We have obtained bounds of the integral operator in different forms. In Theorem 1, upper bounds of this operator are studied for an exponentially convex function and several 
special cases have been presented in the form of propositions and corollaries. The boundedness is studied in Theorem 2. In Theorem 3, we have obtained results for differentiable function $f$ such that $\left|f^{\prime}\right|$ is exponentially convex. A version of the Hadamard inequality is proved in Theorem 4 which leads to its several variants for fractional and conformable integral operators.

\section{Data Availability}

No data were used to support this article.

\section{Conflicts of Interest}

The authors declare that they have no conflicts of interest.

\section{Acknowledgments}

This work was supported in part by the Basic Public Welfare Research Projects in Zhejiang Province (LGG 19F050003).

\section{References}

[1] C. P. Niculescu and L. E. Persson, "Convex functions and their applications-a contemporary approach,"CMS Books in Mathematics, vol. 23, Springer Verlag, New York, NY, USA, 2006.

[2] J. Pečarić, F. Proschan, and Y. L. Tong, Convex Functions, Partial Orderings, and Statistical Applications, Academics Press, New York, NY, USA, 1992.

[3] A. W. Roberts and D. E. Varberg, Convex Functions, Academic Press, New York, NY, USA, 1973.

[4] M. U. Awan, M. A. Noor, and K. I. Noor, "Hermite-Hadamard inequalities for exponentially convex functions," Applied Mathematics \& Information Sciences, vol. 12, no. 2, pp. 405-409, 2018.

[5] Y. C. Kwun, G. Farid, S. M. Kang, B. K. Bangash, and S. Ullah, "Derivation of Bounds of several kinds of operators via $(s, m)$-convexity," Advances in Difference Equations, vol. 2020, p. 5, 2020.

[6] G. Alirezaei and R. Mathar, "On exponentially concave functions and their impact in information theory," in Proceedings of the 2018 Information Theory and Applications Workshop, San Diego, CA, USA, 2018.

[7] S. S. Dragomir and I. Gomm, "Some Hermite-Hadamard type inequalities for functions whose exponentials are convex," Studia Universitatis Babeş-Bolyai Mathematica, vol. 60, no. 4, pp. 527-534, 2015.

[8] M. A. Noor and K. I. Noor, "Strongly exponentially convex functions," University Politehnica of Bucharest Scientific Bulletin-Series A-Applied Mathematics and Physics, vol. 81-82, 2019.

[9] S. Pal and T. K. Wong, "On exponentially concave functions and a new information geometry," Annals of Probability, vol. 2, no. 46, pp. 1070-1113, 2018.

[10] J. Pečarić, I. Perić, and G. Roqia, "Exponentially convex functions generated by Wulbert's inequality and Stolarskytype means," Mathematical and Computer Modelling, vol. 55, pp. 1849-1857, 2012.

[11] S. Rashid, T. Abdeljawad, F. Jarad, and M. A. Noor, "Some estimates for generalized Riemann-Liouville fractional integrals of exponentially convex functions and their applications," Mathematics, vol. 7, no. 9, p. 807, 2019.
[12] S. Rashid, M. A. Noor, and K. I. Noor, "Fractional exponentially $\mathrm{m}$-convex functions and inequalities," International Journal of Analysis and Applications, vol. 3, no. 17, pp. 464478, 2019.

[13] S. Rashid, M. A. Noor, and K. I. Noor, "New estimates for exponentially convex functions via conformable fractional operator," Fractal and Fractional, vol. 3, no. 2, p. 19, 2019.

[14] S. Rashid, M. A. Noor, and K. I. Noor, "Modified exponential convex functions and inequalities," Open Access Journal of Mathematical and Theoretical Physics, vol. 2, no. 2, pp. 45-51, 2019.

[15] S. Mubeen and G. M. Habibullah, " $k$-fractional integrals and applications," International Journal of Contemporary Mathematical Sciences, vol. 7, no. 2, pp. 89-94, 2012.

[16] A. A. Kilbas, H. M. Srivastava, and J. J. Trujillo, "Theory and applications of fractional differential equations,"North-olland Mathematics Studies, vol. 204, Elsevier, Amsterdam, Netherlands, 2006.

[17] G. Farid, "Existence of an integral operator and its consequences in fractional and conformable integrals," Open Journal of Mathematical Sciences, vol. 3, no. 1, pp. 210-216, 2019.

[18] H. Chen and U. N. Katugampola, "Hermite-Hadamard and Hermite-Hadamard-Fejér type inequalities for generalized fractional integrals," Journal of Mathematical Analysis and Applications, vol. 446, no. 2, pp. 1274-1291, 2017.

[19] S. S. Dragomir, “Inequalities of Jensen's type for generalized $k-g$-fractional integrals of functions for which the composite $f \circ g^{-1}$ is convex," Fractional Differential Calculus, vol. 20, pp. 127-150, 2017.

[20] S. Habib, S. Mubeen, and M. N. Naeem, "Chebyshev type integral inequalities for generalized $k$-fractional conformable integrals," Journal of Inequalities and Special Functions, vol. 9, no. 4, pp. 53-65, 2018.

[21] F. Jarad, E. Ugurlu, T. Abdeljawad, and D. Baleanu, "On a new class of fractional operators," Advances in Difference Equations, vol. 2017, no. 1, p. 247, 2017.

[22] T. U. Khan and M. A. Khan, "Generalized conformable fractional operators," Journal of Computational and Applied Mathematics, vol. 346, pp. 378-389, 2019.

[23] A. A. Kilbas, O. I. Marichev, and S. G. Samko, Fractional Integrals and Derivatives; Theory and Applications, Gordon \& Breach, Lausanne, Switzerland, 1993.

[24] M. Z. Sarikaya, M. Dahmani, M. E. Kiris, and F. Ahmad, " $(k, s)$-Riemann-Liouville fractional integral and applications," Hacettepe Journal of Mathematics and Statistics, vol. 45, no. 1, pp. 77-89, 2016.

[25] T. Tunc, H. Budak, F. Usta, and M. Z. Sarikaya, "On new generalized fractional integral operators and related fractional inequalities," 2017, https:/www.researchgate.net/publication/ 313650587.

[26] G. Farid, W. Nazeer, M. Saleem, S. Mehmood, and S. Kang, "Bounds of Riemann-Liouville fractional integrals in general form via convex functions and their Applications," Mathematics, vol. 6, no. 11, p. 248, 2018.

[27] G. Farid, "Some Riemann-Liouville fractional integral for inequalities for convex functions," The Journal of Analysis, vol. 27, no. 4, pp. 1095-1102, 2018.

[28] L. N. Mishra, G. Farid, and B. K. Bangash, "Bounds of an integral operator for convex functions and results in fractional calculus," Honam Mathematical Journal, vol. 42, no. 2, pp. 359-376, 2020. 
[29] M. Z. Sarikaya and F. Ertuğral, "On the generalized HermiteHadamard inequalities,” 2017, https://www.researchgate.net/ publication/321760443.

[30] G. Hong, G. Farid, J. Pečarić et al., "Boundedness of fractional integral operators containing Mittag-Leffler function via exponentially convex functions," Journal of Mathematics, vol. 2020, Article ID 3584105, 7 pages, 2020. 